\title{
Investigation of Microgrids with Both Inverter Interfaced and Direct AC Connected Distributed Energy Resources
}

\author{
Zhixin Miao, Senior Member, IEEE, Alexander Domijan, Lingling Fan, Senior Member, IEEE
}

\begin{abstract}
With the increasing usage of renewable energy resources and energy storage devices, inverter-based distributed energy resources (DERs) become the important components in microgrids. As diesel generators with direct ac connections are the current most cost effective and reliable power sources, the stability investigation of microgrids should include both types of DERs. In this paper, dynamics of diesel generation will be included and the interaction of the diesel generators and the inverter-based DERs will be investigated using eigenvalue analysis and time-domain simulations. The significant contributions of this paper include: (i) identification of the stability problem in microgrids with both inverter-based DERs and conventional generators and (ii) investigation of the interaction problem of inverter-based DERs and conventional generators in islanded microgrids.
\end{abstract}

Index Terms-Microgrid, Stability, Diesel Engine Generator, Inverter, Droop

\section{INTRODUCTION}

$\mathbf{M}$ ORE and more inverter-based distributed energy resources (DERs) using renewable energy (wind and solar) or micro-sources (fuel cells and micro-turbine) are put into use nowadays. The stability of microgrids with inverter interfaced DERs has been investigated in [1]-[3]. It is found that frequency droop parameters have impact on system stability. Diesel generators with direct ac connections are the most cost effective, reliable and popular distributed generation [4]. Meanwhile, there is a need of backup energy sources due to the intermittent nature of renewable energy sources such as solar and wind. Diesel generators have been used as the backup in a CERTS microgrid [5]. In some other scenarios, diesel generators are still the main source while wind/solar are put into use to reduce the consumption of fossil fuel or to keep a high power quality. Therefore, the stability investigation should include both inverter-based DERs and direct ac connection based DERs. Such research topic will be the focus of this paper.

In this research, linear system analysis based approaches will be adopted for stability investigation of microgrids with both types of DERs. Since frequency and voltage droops have been found to have significant impact on stability, the following paragraphs give detailed description of active and reactive power sharing.

Active Power Sharing

Under grid-connected conditions, inverter-based DERs in a

Z. Miao, A. Domijan and L. Fan are with Dept. of Electrical Engineering, University of South Florida, Tampa, FL 33620. Email: zhixin.miao@ieee.org. microgrid are in power flow control mode to follow the active and reactive power orders. Under islanded conditions, inverterbased DERs are operated in the frequency-power $(f-P)$ and voltage-reactive power $(V-Q)$ droop modes to participate in the active and reactive power sharing [1], [6]. The purpose of the droops is to keep power balance and to regulate the frequency and the voltage in the microgrid. The $f-P$ droop is very similar to the frequency droop used in the speed governor of synchronous generators [7]. When the system has a load increase, kinetic energy from the rotating masses of the synchronous generators will be released which causes the rotating speed to drop. The droop control in the speed governors guarantees the increase of the outputs from the prime movers to balance the load demand.

Reactive Power Sharing

Under islanded conditions, inverter-based DERs also have the responsibility to regulate voltages. Voltage-reactive power or $V-Q$ droop control method is popularly applied in microgrids [6]. In addition, voltage droop control can be achieved through reactive current instead of reactive power [8].

Stability Issues in Microgrids

Small signal stability of microgrids under islanded conditions is an important concern and has been investigated in [1]-[3]. Parameters that affect stability include frequency droop gains and the dominant dynamics have low oscillation frequency due to the droop control loops [1]. Quantitative analysis is usually given by eigenvalue analysis. In order to improve the damping, the droop control loops are supplemented with the differential of power $(d P / d t$ in frequency droop and $d Q / d t$ in voltage droops) [2]. Moreover computational methods to determine the stability of a multi-inverter microgrid are presented in [9]. In [10], quantitative analysis is given to compute the distance to instability in a multi-dimension parameter space. In [11], a new droop control method relying on communication is proposed and tested to be able to operate stably for a range of operating conditions while ensuring satisfactory load sharing. Alternatively, instead of $P-f$ control, angle droop control $(\mathrm{P}-\delta)$ is used where machine angle is used as measurement in droop control [12].

The aforementioned research focuses on microgrids with inverter-based DERs only. Since diesel generator is an important component in microgrids, the stability investigation of microgrids with both inverter-based DERs and diesel generators is of significant importance. Two recent papers have investigated dynamic performance of microgrids with both types of DERs. Reference [13] gives time-domain simulation 
results of a renewable energy power generation system which includes three wind turbine generators (WTGs), a diesel engine generator, two fuel cells (FCs), and a photovoltaic system (PV) while the energy storage subsystems consist of a battery energy storage system and a flywheel energy storage system. Reference [14] investigates the dynamic performance a microgrid with two DERs at islanding transient periods. One DER is a conventional synchronous machine and the other is inverter-interfaced. The simulation studies based on PSCAD/EMTDC show that an appropriate control strategy for the inverter-interfaced DER can ensure stability of the microgrid and maintain voltage quality at designated buses.

Both [13] and [14] are based on simulation studies. They do not provide analytical results related with system modes and sensitivity analysis of controller parameters. As a step further, Reference [15] documents the linear models of a microgrid with both synchronous generator and voltage source inverter interfaced DERs. Impact of DER power/reactive power dispatch level, voltage and power control loop parameters, and the phase locked loop (PLL) parameters on the system small signal stability has been discussed. However, the impact of frequency and voltage droops on stability is not discussed and diesel generators are not specifically studied.

Diesel generator is one focus of this research. Microgrids with both diesel and inverter-based DERs show frequency oscillations due to a diesel engine in a CERT microgrid [5]. Small signal analysis of the interactions of diesel generators and inverter-based DERs will reveal more about the characteristics of such systems.

Therefore, the objective of this research is two-fold: (i) to investigate the stability of a microgrid with both types of DERs (with or without inverter interfaces) under islanded conditions and (ii) to investigate the interactions between the inverterbased DERs and the diesel generators. Unlike the investigation conducted in [1], [2] where only inverter-based DERs are taken into consideration, in this investigation, diesel generators will be modeled and included in the system.

\section{STUDY SYSTEM}

The study system is shown in Fig. 1. The system is built based on the benchmark system of IEEE standard 399-1997 [16]. The parameters of the system are given in Appendix. Two diesel engine generators and two converter-interfaced DERs form a microgrid. Under islanded conditions, the breaker connected to the utility is open.

\section{A. Diesel Generator}

Diesel is used as fuel to produce heat and pressure to move a piston inside a cylinder, then this linear motion is converted to rotation of a crankshaft, which spin an AC electric generator. A diesel generator used in distribution systems can have a size from $5 \mathrm{~kW}$ to $25 \mathrm{MW}$ [4] (Chapter 6). AC frequency is controlled by controlling the engine speed [4]. The diesel generators in this research are modeled as synchronous generators with excitation systems and governorturbines.

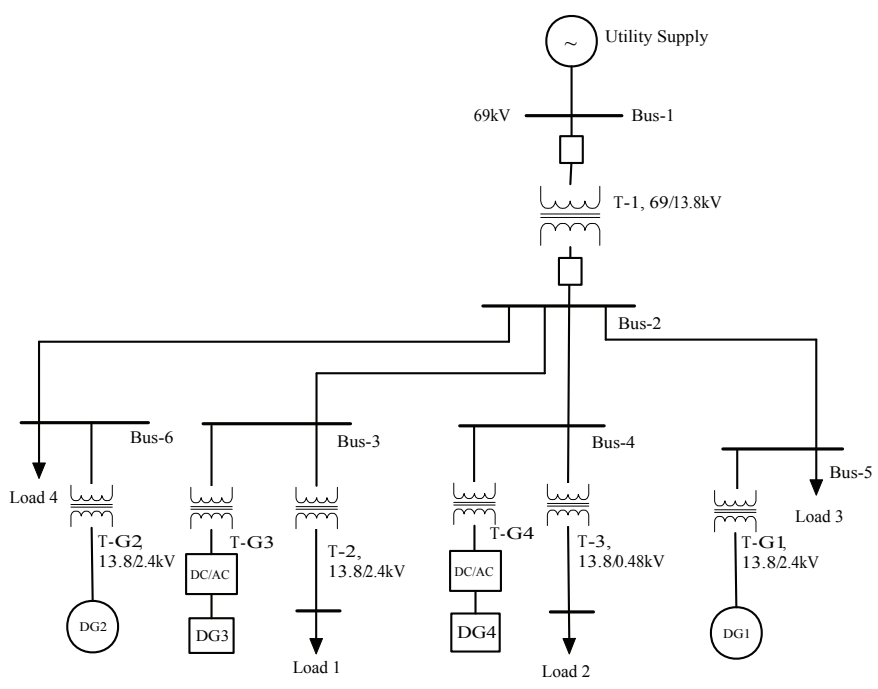

Fig. 1. IEEE Distribution System Test System.

The dynamic model of a $2.4 \mathrm{kV} 3.125$ MVA diesel generator is developed in [17] where the generator is a salient pole synchronous generator. The diesel generator is equipped with an excitation system to control the terminal voltage. Models of the synchronous generator, excitation system and governor are given along with parameters. In [18], a diesel engine driven permanent magnet synchronous generator is modeled. The model used in this paper is derived based on models in [17], [18].

1) Synchronous Generator: A subtransient synchronous generator model including amortisseur effect is used in this paper. The details of the model can be found in [19].

2) Governor Model: Diagram of the diesel engine and the governor is presented in Fig. 2. The frequency droop compares the speed of the synchronous generator with the reference value and a gain $K_{D T}$ is specified. The output of the frequency droop modifies the reference value of the fuel input. The governor system and the actuator are modeled by two secondorder transfer functions. A most significant feature of a diesel engine is its delay block [5]. The parameters in Fig. 2 are listed in Appendix.

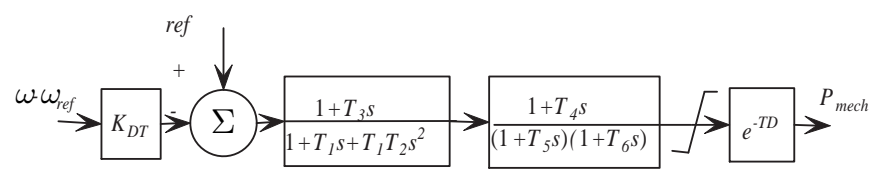

Fig. 2. Diesel engine and governor model.

3) Excitation System Model: To maintain a constant terminal voltage, automatic voltage regulator is used to adjust the excitation current in the rotor winding of the synchronous generator. The block diagram of the excitation system is shown in Fig. 3. The parameters are listed in Appendix.

\section{B. Inverter-based DERs}

Inverter-based DERs can be simply regarded as constant dc voltage sources connected to three-phase inverters. The 


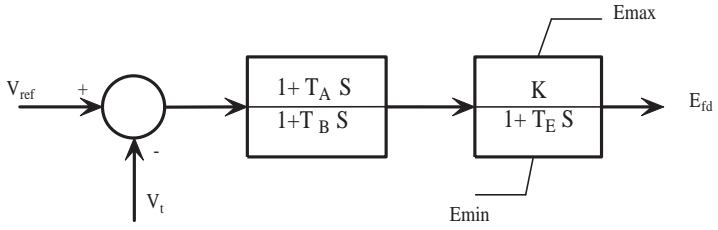

Fig. 3. Excitation system model.

schematic connection of a voltage source inverter (VSC) is shown in Fig. 4 which consists of six IGBT switches driven by PWM gate circuits. The VSC converts dc voltage into threephase ac voltage.

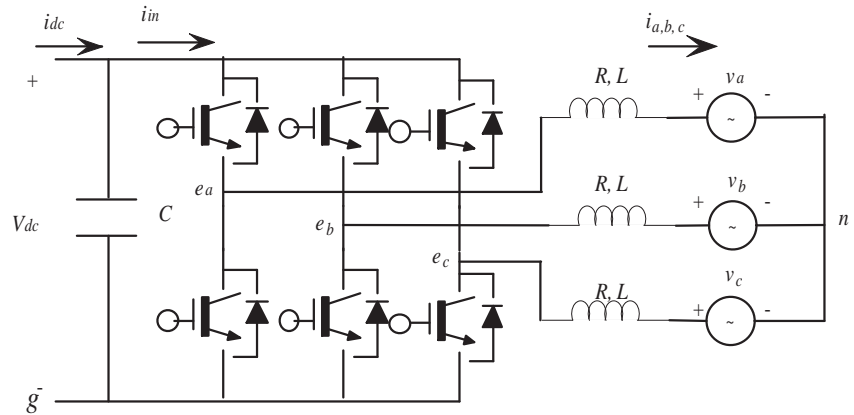

Fig. 4. Schematic diagram of a VSC inverter.

\section{VSC Model}

VSCs are used to interface the DERs to the microgrid. To analyze the dynamic performance of the inverter-based DER, the average three-phase DC-AC VSC model including the aclink inductor dynamics and the dc-link capacitor dynamics is first developed in the instantaneous $a b c$ frame applying KCL and KVL:

$$
\left\{\begin{array}{l}
L \frac{d i_{a}}{d t}=-R i_{a}-v_{a n}+e_{a n} \\
L \frac{d i_{b}}{d t}=-R i_{b}-v_{b n}+e_{b n} \\
L \frac{d i_{c}}{d t}=-R i_{c}-v_{c n}+e_{c n} \\
C \frac{d V_{d c}}{d t}=i_{i n}-i_{d c}
\end{array}\right.
$$

where $i_{i n}, i_{d c}, i_{a, b, c}, v_{a, b, c n}$ and $e_{a, b, c n}$ are all labeled in Fig. 4. To relate the model in (1) to the duty cycle of the PWM switching of the converter, the exporting dc current $i_{i n}$ and the output voltages of the inverter $e_{a, b, c n}$ will be reexamined.

Neglecting the switching loss of the inverter, the instantaneous power coming to the inverter from the dc side $\left(P_{d c}\right)$ should equal to the power transferring to the ac side $\left(P_{a c}\right)$. The following equations are given:

$$
\left\{\begin{array}{l}
P_{a c}=P_{d c} \\
P_{a c}=v_{a} i_{a}+v_{b} i_{b}+v_{c} i_{c} . \\
P_{d c}=V_{d c} i_{i n} .
\end{array}\right.
$$

Hence the expression of the dc side current $i_{i n}$ can be expressed as

$$
i_{i n}=\frac{v_{a} i_{a}+v_{b} i_{b}+v_{c} i_{c}}{V_{d c}}=\sum_{i=a, b, c} i_{i} d_{i}
$$

where $d_{a, b, c}$ are the duty cycles of the PWM and $d_{i}=\frac{v_{i}}{V_{d c}}, i=$ $a, b, c$.

The three-phase voltages from the VSC can be expressed as:

$$
\left\{\begin{array}{l}
e_{a n}=e_{a g}-v_{n g}=d_{a} V_{d c}-v_{n g} \\
e_{b n}=e_{b g}-v_{n g}=d_{b} V_{d c}-v_{n g} \\
e_{c n}=e_{a g}-v_{n g}=d_{c} V_{d c}-v_{n g}
\end{array} .\right.
$$

Considering a three-wire connected balanced AC system where the summation of the three-phase voltages is zero: $v_{a n}+v_{b n}+v_{c n}=0$, then the neutral to ground voltage $v_{n g}$ can be expressed as follows.

$$
v_{n g}=\frac{e_{a g}+e_{b g}+e_{c g}}{3}=\sum_{i=a, b, c} d_{i} \frac{V_{d c}}{3} .
$$

Hence the model in (1) can be expressed as:

$$
\left\{\begin{array}{l}
L \frac{d i_{a}}{d t}=-R i_{a}-v_{a n}+V_{d c}\left(d_{a}-\frac{\sum_{i=a, b, c} d_{i}}{3}\right) \\
L \frac{d i_{b}}{d t}=-R i_{b}-v_{b n}+V_{d c}\left(d_{b}-\frac{\sum_{i=a, b, c} d_{i}}{3}\right) \\
L \frac{d i_{c}}{d t}=-R i_{c}-v_{c n}+V_{d c}\left(d_{c}-\frac{\sum_{i=a, b, c} d_{i}}{3}\right) \\
C \frac{d V_{d c}}{d t}=\sum_{i=a, b, c} i_{i} d_{i}-i_{d c}
\end{array}\right.
$$

The mathematic model above concurs with the one used in [20]. Our paper gives a detailed derivation procedure to help understanding the model. The $q d$ reference frame where $d$-axis leads the $q$-axis by $90^{\circ}$ is widely used in modeling inverterbased circuits. Therefore, the model in (6) will eventually be transformed into a $q d$ reference frame. All three-phase variables (e.g., voltages, currents, switch functions) can be transformed to a $q d$ reference frame by the $q d / a b c$ relationship.

$$
\left[\begin{array}{c}
f_{q} \\
-f_{d} \\
f_{0}
\end{array}\right]=K_{s}\left[\begin{array}{l}
f_{a} \\
f_{b} \\
f_{c}
\end{array}\right]
$$

where $K_{s}=\frac{2}{3}\left[\begin{array}{ccc}\cos \theta & \cos \left(\theta-\frac{2 \pi}{3}\right) & \cos \left(\theta+\frac{2 \pi}{3}\right) \\ \sin \theta & \sin \left(\theta-\frac{2 \pi}{3}\right) & \sin \left(\theta+\frac{2 \pi}{3}\right) \\ \frac{1}{2} & \frac{1}{2} & \frac{1}{2}\end{array}\right]$

where $\theta$ is an arbitrary angle. If $\theta=\int \omega_{s} d t$ where $\omega_{s}$ is the synchronous frame's angular frequency, then the $q d$ reference frame is a synchronous reference frame.

The model in (6) in a synchronous reference frame is given as:

$$
\left\{\begin{array}{l}
L \frac{d i_{q}}{d t}=-R i_{q}+\omega L i_{d}+v_{q}-V_{d c} d_{q} \\
L \frac{d i_{d}}{d t}=-R i_{d}-\omega L i_{q}+v_{d}-V_{d c} d_{d} \\
C \frac{d V_{d c}}{d t}=\frac{3}{2}\left(d_{q} i_{q}+d_{d} i_{d}\right)-i_{d c}
\end{array}\right.
$$

Since the inverter-based DERs are integrated into an ac microgrid, per unit system is preferred. The RL circuit model at the ac side in (9) can be expressed in state-space and in per unit system as listed in (10).

$\frac{d}{d t}\left[\begin{array}{c}i_{q} \\ i_{d}\end{array}\right]=\omega\left[\begin{array}{cc}-\frac{R}{X} & 1 \\ -1 & -\frac{R}{X}\end{array}\right]\left[\begin{array}{c}i_{q} \\ i_{d}\end{array}\right]+\frac{\omega}{X}\left[\begin{array}{c}v_{q}-V_{d c} d_{q} \\ v_{d}-V_{d c} d_{d}\end{array}\right]$

where all currents, voltages and impedances are per unit values and $\omega=377 \mathrm{rad} / \mathrm{s}$. 


\section{Inner Current Control Loop}

Vector control is popularly used in converter control where the $q$-axis is aligned to the output voltage vector. Hence, $V_{q}=$ $V$, and $V_{d}=0$. Thus (9) can be expressed as:

$$
\left\{\begin{array}{l}
V=-L \frac{d i_{q}}{d t}-R i_{q}+\omega L i_{d}-V_{d c} d_{q} \\
0=-L \frac{d i_{d}}{d t}-R i_{d}-\omega L i_{q}-V_{d c} d_{d}
\end{array}\right.
$$

The cross coupling terms in (11) can be treated as disturbance and new variables are defined to have the following relationship [21]:

$$
\left\{\begin{array}{l}
v_{q}^{\prime}=R i_{q}+L \frac{d i_{q}}{d t} \\
v_{d}^{\prime}=R i_{d}+L \frac{d i_{d}}{d t}
\end{array}\right.
$$

Equation (12) indicates that the first-order dynamics of $i_{d}$ and $i_{q}$ are determined by $v_{q}^{\prime}$ and $v_{d}^{\prime}$. Proportional and integral (PI) control schemes are widely adopted in such scenarios to make the currents track their reference values. The relationship of $v_{q}^{\prime}, v_{d}^{\prime}$ and the output voltages from the inverter $\left(d_{q} V_{d c}\right.$ and $d_{d} V_{d c}$ ) can be expressed as follows:

$$
\left\{\begin{array}{l}
V_{d c} d_{q}=v_{q}^{\prime}+\omega L i_{d}+V \\
V_{d c} d_{d}=v_{d}^{\prime}-\omega L i_{q}+0
\end{array}\right.
$$

Equation (13) describes the control law of the inverter's inner current loop.

\section{E. Outer Power Loop}

The alignment of the voltage vector to the $q$-axis also simplifies active power and reactive power expression [21]. The active power will be proportional to $i_{q}$ while the reactive power will be proportional to $i_{d}$. The overall control diagram of inverters is shown in Fig. 5 to provide load following service. The control loops consist of inner fast current control

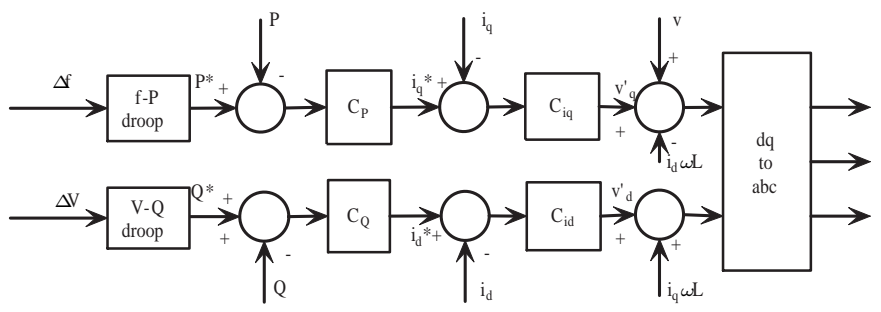

Fig. 5. Control diagram of an inverter.

loops and outer slow power control loops. The reference active/reactive powers are obtained through the $f-P$ droop characteristic which is shown in Fig. 6. Proportional and integral (PI) controllers are used in the inverter controls. The outer power control loops generate reference currents $i_{q}^{*}$ and $i_{d}^{*}$. The inner current controls which are PI controls will force the measurements $i_{q}$ and $i_{d}$ to follow the reference values.

The droop characteristics for active/reactive power sharing are shown in Fig. 6. The slopes are defined as droops: $R$ for the $f-P$ slope and $R_{V Q}$ for the $V_{Q}$ slope. When the system has a decrease in frequency $(\Delta f)$, each DER equipped with $f-P$ droop will act to increase its output power according to its $f-P$ characteristics. Assume the slope of $f-P$ characteristic

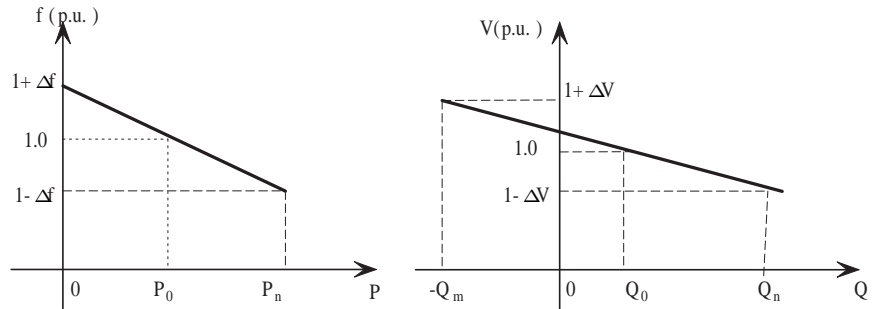

Fig. 6. Droop characteristics for active/reactive power sharing among DERs: a) $f-P$ droop, b) $V-Q$ droop.

of $\mathrm{DER}_{i}$ is $R_{i}$, then the total change of the DER exporting power should be

$$
\Delta P=-\sum_{i} \frac{1}{R_{i}} \Delta f=\Delta P_{D}
$$

Hence, each DER will take its share of the load increase and the share is expressed as follows

$$
\text { share }_{i}=\frac{\frac{1}{R_{i}}}{\sum_{i} \frac{1}{R_{i}}} .
$$

Similarly, reactive sharing of each DER can be computed given the $V-Q$ slope $R_{V Q i}$ of each DER.

$$
\text { share }_{i}=\frac{\frac{1}{R_{V Q i}}}{\sum_{i} \frac{1}{R_{V Q i}}} .
$$

The Matlab/Simulink block is shown in Appendix. In the Simulink blocks, $K_{f p}=\frac{1}{R}$ and $K_{V Q}=\frac{1}{R_{V Q}}$.

\section{F. System Level Model}

The dynamics of the RL circuits are all ignored in this paper due to their fast responses. Hence phasor-based circuit model is built to represent the system. Each DER is represented by a current source $\bar{I}_{i}=i_{q i}+j i_{d i}$. For the inverter-based DERs, each inverter should have its quantities expressed upon its own synchronous reference frame. One of the $q d$ reference frames is chosen as the system reference frame. While for the synchronous generators, the reference frame is fixed on their rotors. Hence there exist angles between the rotor reference frame, each individual DER reference frame and the system synchronous reference frame.

In order to establish the network equation, all current sources should be expressed in the same reference frame. The synchronous reference frame is chosen as the universal reference frame. Hence a relationship between the voltage phasors and the current phasors is established as follows:

$$
\left[\begin{array}{c}
\bar{V}_{1} \\
\bar{V}_{2} \\
\bar{V}_{3} \\
\bar{V}_{4}
\end{array}\right]=Y^{-1}\left[\begin{array}{c}
\bar{I}_{1} \\
\bar{I}_{2} \\
\bar{I}_{3} \\
\bar{I}_{4}
\end{array}\right]
$$

where $Y$ is the system admittance matrix, $\bar{I}_{1}=\left(i_{q 1}+\right.$ $\left.j i_{d 1}\right) e^{j \delta_{1}}, \bar{I}_{2}=\left(i_{q 2}+j i_{d 2}\right) e^{j \delta_{2}}, \bar{I}_{3}=i_{q 3}+j i_{d 3}$ and $\bar{I}_{4}=i_{q 4}+j i_{d 4}$.

The system level model building technique has been applied in [22] to develop distribution system models. The purpose of 


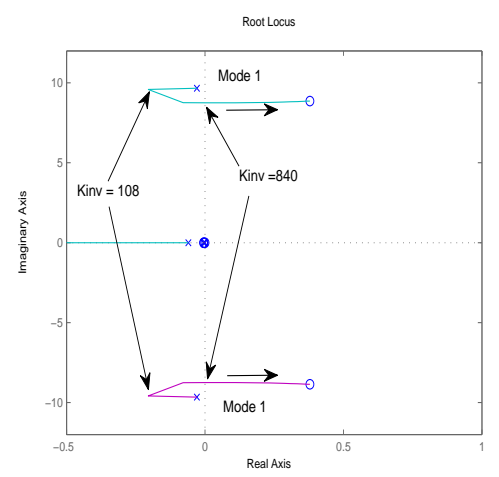

(a) $K_{D T}=0$

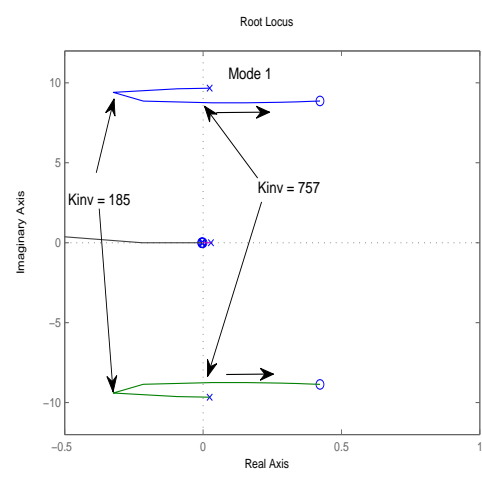

(b) $K_{D T}=1$

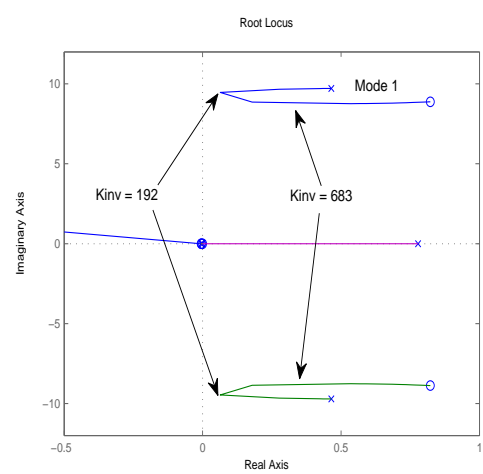

(c) $K_{D T}=10$

Fig. 7. Root locus with a changing $f-P$ droop gain in DER3 of Case 3. The gain of the $f-P$ droop in the diesel generators $K_{D T}$ is specified for each plot.

the system level equation is to find the voltage phasor at each bus. These voltage phasors will then be decomposed into $q d$ axis voltages and used in each DER's dynamic block for next step integration of the $q d$-axis currents. With the system level equation, all DER building blocks are interconnected and a Matlab/Simulink model can be established. The system level Simulink diagram is presented in Appendix. It can be seen that each DER is represented by a block with currents as the outputs. The network block collects the currents from each DER and computes the voltage phasors. These voltage phasors are fed back to each DER block as the inputs. Inside each DER's building block, differential equations are represented by Simulink blocks.

\section{Eigenvalue Analysis}

The system expressed in the synchronous reference frame is built in MATLAB/Simulink for small signal analysis and time-domain simulations using the model described in Section II. The eigenvalue analysis is carried out based on the linear systems derived from the nonlinear dynamic models. The derivation however is carried out by Matlab automatically using small perturbation method and function "linmod". Hence, the linearized model is not provided in this paper.

\section{A. System Modes}

Dominating oscillation modes in the system will be identified and impact of various parameters such as penetration level of inverter-based DERs, the gain of the power-frequency droops of two kinds of DERs will be discussed in this section. Three operating scenarios are listed in Table I. The total demand is $10 \mathrm{MW}$.

TABLE I

POWER EXPorTING LEVELS FROM EACH POWER SOURCE

\begin{tabular}{||l|c|c|c|c||}
\hline \hline & Diesel1 & Diesel2 & DG3 & DG4 \\
\hline Case 0 & $5 \mathrm{MW}$ & $5 \mathrm{MW}$ & 0MW & 0MW \\
\hline Case 1 & $3.5 \mathrm{MW}$ & $3.5 \mathrm{MW}$ & $1.5 \mathrm{MW}$ & $1.5 \mathrm{MW}$ \\
\hline Case 2 & $3 \mathrm{MW}$ & $3 \mathrm{MW}$ & $2 \mathrm{MW}$ & $2 \mathrm{MW}$ \\
\hline Case 3 & $2 \mathrm{MW}$ & $2 \mathrm{MW}$ & $3 \mathrm{MW}$ & $3 \mathrm{MW}$ \\
\hline Case 4 & $0 \mathrm{MW}$ & $0 \mathrm{MW}$ & $5 \mathrm{MW}$ & $5 \mathrm{MW}$ \\
\hline \hline
\end{tabular}

The dominant system modes are identified in Table II. Mode 1 is identified to be the electromechanical mode of the diesel generators. The frequency of the mode is about $1.5 \mathrm{~Hz}(9$ $\mathrm{rad} / \mathrm{s})$. Mode 2 and 3 are identified to be related to the outer power control loops of inverters. While Mode 2 is related to the $f-P$ droop proportional gain $K_{p 1}$ in the inverters, Mode 3 is related to the integral gain $K_{i 1}$.

TABLE II

SYSTEM MODES

\begin{tabular}{||c|c|c|c||}
\hline \hline Scenario & Mode 1 & Mode 2 & Mode 3 \\
\hline Case 0 & $-0.105 \pm j 9.70$ & $-5.63 \pm j 12.44$ & $-1.39 \pm j 1.6$ \\
\hline Case 1 & $-0.111 \pm j 9.54$ & $-5.6 \pm j 12.6$ & $-1.53 \pm j 2.2$ \\
\hline Case 2 & $-0.085 \pm j 9.51$ & $-5.6 \pm j 12.6$ & $-1.58 \pm j 2.2$ \\
\hline Case 3 & $-0.0467 \pm j 9.47$ & $-5.6 \pm j 12.6$ & $-1.69 \pm j 2.2$ \\
\hline Case 4 & $-0.0455 \pm j 9.58$ & $-5.4 \pm j 12.71$ & $-2.10 \pm j 1.9$ \\
\hline
\end{tabular}

From Table II, it is found that with the increasing penetration of the inverter-based DERs, the damping of the dominating system mode (diesel generator electromechanical mode) decreases, however the change of the damping is limited in the range of $(0.47 \%-1.16 \%)$.

In the following paragraphs, impact of the frequency-power droop on the dominating system mode will be investigated.

\section{B. Effect of $f$-P droop on dominating system modes}

The root locus of a changing $f-P$ droop gain in one of the inverter-based DERs is shown in Fig. 7.

From the root locus diagram in Fig. 7, it is found that the gain of the $f-P$ droops in the inverter-based DERs do not impact the system stability significantly. Rather, the gain of the $f-P$ droop in the diesel engine impacts system stability significantly. A larger gain means more participation in load sharing. In Fig. 7, it is noted that the larger the gain, or the more the diesel engines participate into load sharing, the more unstable the microgrid becomes. This phenomenon is verified by the root locus diagram when the $f-P$ droop in the diesel engine is disconnected and the turbine input and the generator speed are treated as the input and output of an open-loop plant. The root locus diagram is shown in Fig. 8. When the gain reaches 1.5 , the microgrid goes unstable from the root 
locus diagram. Therefore, the diesel generators should have a limited gain. This results in an insignificant participation in load sharing.

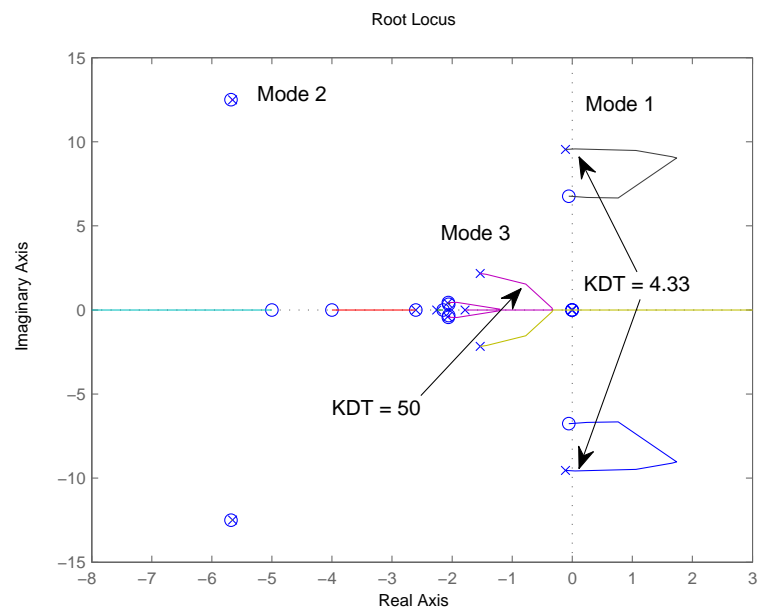

Fig. 8. Root locus with a changing $f-P$ droop gain in the diesel of Case 3 . The gain of the $f-P$ droop of the inverters $K_{i n v}=25$.

\section{Effect of $V-Q$ droop on dominating system modes}

The root locus diagrams of a changing $V-Q$ droop gain in DG3 are shown in Fig. 9. It is found that for the dominating system mode, its pole and zero are very close. Therefore, $V-$ $Q$ droop has negligible impact on this system mode. However, increasing the gain causes another mode to move to the right half plane. The gain should be limited to 6.46 according to the root locus diagram. Table III lists the two system modes under different $V-Q$ droop gain. It is found that Mode 2 experience significant changes in both damping and frequency.

TABLE III

System MOdES For A CHANGING $V-Q$ GAIN IN CASE 3

\begin{tabular}{||c|c|c||}
\hline \hline Gain & Mode 1 & Mode 2 \\
\hline 1 & $-0.0467 \pm j 9.47$ & $-5.2856 \pm j 12.97$ \\
\hline 6 & $-0.0479 \pm j 9.47$ & $-1.1913 \pm j 15.29$ \\
\hline 10 & $-0.0578 \pm j 9.47$ & $1.2949 \pm j 5.6552$ \\
\hline \hline
\end{tabular}

Remarks from the above eigenvalue analysis can be summarized as follows.

1) The diesel generator electromechanical mode is most vulnerable mode - this is due to the time lag of diesel engine governor;

2) the frequency-power droop gain of the diesel engine has a significant impact on the mode. The less the diesel engine participates into load sharing, the more stable the microgrid;

3) the frequency-power $(f-P)$ droop gain of the inverterbased DER has insignificant impact on the electromechanical mode.

4) the voltage-reactive power $(V-Q)$ droop gain of the inverter-based DER has a negligible impact on the electromechanical mode. However, it has a significant impact on Mode 2 and the gain should not exceed the limit to keep the microgrid stable.

\section{Time-Domain Simulation Results}

Time domain simulations are carried out in Matlab/Simulink. Simulations are conducted for Case 3 where the penetration level of the inverter-based DERs reaches $60 \%$.

\section{A. Reference Power Step Response}

Step responses are added to the reference power of the two inverter-based DERs to test the effectiveness of their power control loops. A $0.02 \mathrm{pu}$ increase is added to the reference active power of DG3 while a $0.02 \mathrm{pu}$ decrease is added to the reference active power of DG4. Dynamic responses are presented in Fig. 10. It is found that the PI controllers in the inverters effectively bring the active power from DG3 and DG4 to match the step response.

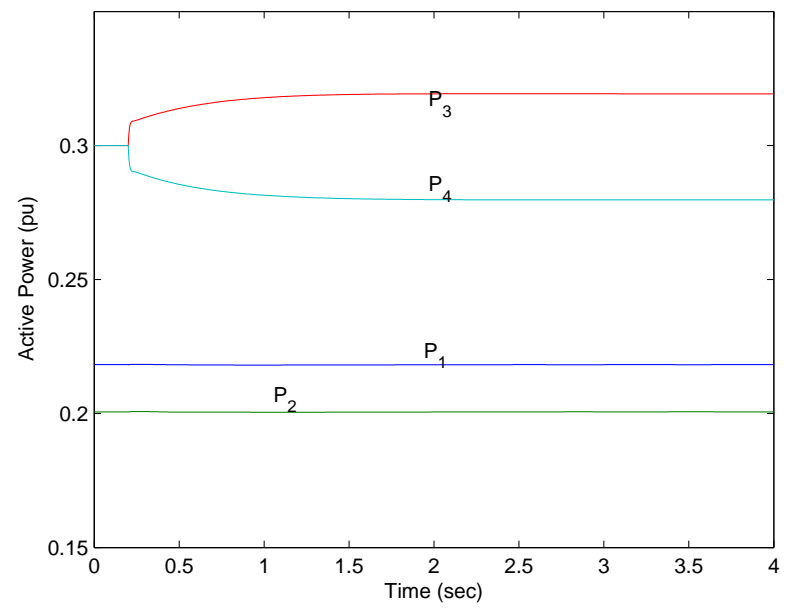

Fig. 10. Dynamic responses of four DERs' outputs under a step change of $0.02 \mathrm{pu}$ increase in the real power order of DER3 and a step change of 0.02 pu decrease in the real power order of DER4. Operating condition: Case 3.

\section{B. Load Sharing}

At $t=4 s$, a $0.034 \mathrm{pu}$ increase is added to the load at Bus 5. The four DERs are expected to pick up the load. The gains of the diesel generators are set to 0.1 while the inverterbased DERs have a gain of 25. Since $K_{i n v}$ is the inverse of the frequency-power slope $R_{i n v}$, the larger the gain, the shallower the slope and the more the DER participates into load sharing. In this case, the majority of the load increase will be picked by DG3 and DG4. The dynamic responses of the four DERs output active powers are shown in Fig. 11. The power of the diesel generators registers an oscillation of about $1.5 \mathrm{~Hz}$. This is the electromechanical mode.

The two inverter sources do not participate at the inception of the load change in Fig. 11. The diesel generators pick up the load change initially and they are brought back to their initial production when the inverter DERs increase their output. This is because for synchronous generators, rotating energy can 


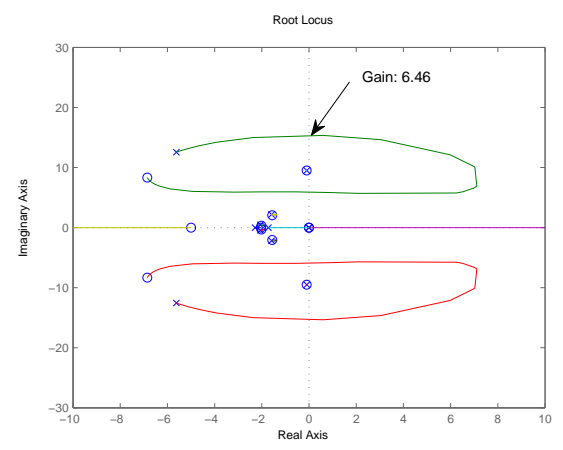

(a)

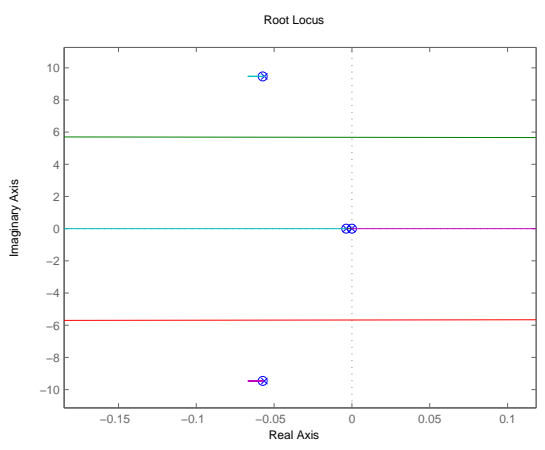

(b)

Fig. 9. Root locus with a changing $V-Q$ droop gain in DER3. Operating condition: Case 3. $K_{D T}=0.1, K_{i n v}=25$. (a) and (b) are both root locus diagram with (b) zoomed in to show the dominant mode.

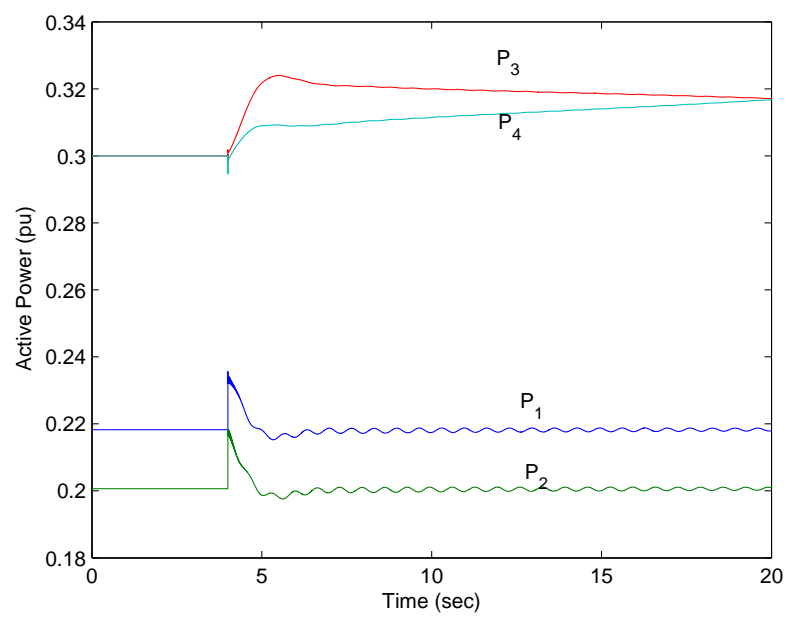

Fig. 11. Dynamic responses of four DERs' outputs when there is an increase $(0.034 \mathrm{pu})$ in the load at Bus 5. Operating condition: Case 3. $K_{i n v}=25$, $K_{D T}=0.1$ where $K_{i n v}$ is the gain of f-P loop in VSC inverters and $K_{D T}$ is the gain of f-P loop in the diesel governor.

be released and converted to electric energy with the rotating speed reduced. However, inverter interfaced DERs even with storage have no such capability unless supplementary inertial response control loop can be designed. The concept of inertial response control for power electronic converter interfaced wind generation has been conveyed in [23].

\section{Impact of Diesel Turbine's f-P Gain}

To verify the impact of the diesel turbine's $f-P$ gain on stability, the gain is increased from 0.1 to 10 and the simulation results are shown in Figs. 12-13. From the simulation results, the sustained oscillation makes the system lose stability. All the powers register the electromechanical oscillation.

The simulation results corroborate with the eigenvalue analysis in Section III.

\section{CONClusion}

The dynamic model of a microgrid with both diesel generator and DERs with inverter interfaces are developed in

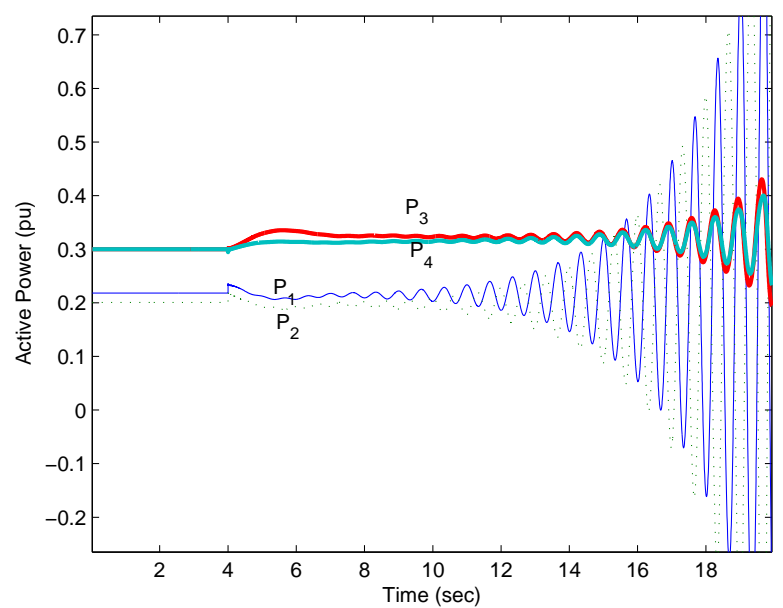

Fig. 12. Dynamic responses of four DERs' outputs when there is an increase $(0.034 \mathrm{pu})$ in the load at Bus 5. Operating condition: Case 3. $K_{\text {inv }}=25$, $K_{D T}=10$ where $K_{i n v}$ is the gain of f-P loop in VSC inverters and $K_{D T}$ is the gain of f-P loop in the diesel governor.

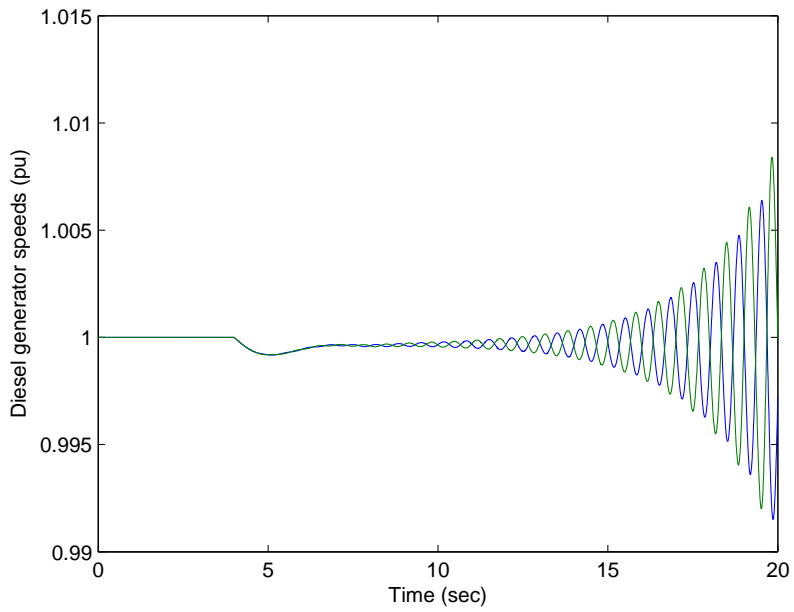

Fig. 13. Dynamic responses of the speeds of the two diesels when there is an increase $(0.034 \mathrm{pu})$ in the load at Bus 5. Operating condition: Case 3. $K_{i n v}=25, K_{D T}=10$ where $K_{i n v}$ is the gain of f-P loop in VSC inverters and $K_{D T}$ is the gain of f-P loop in the diesel governor. 
Matlab/Simulink. A study system with both diesel generators and inverter-based DERs is built. Interactions of DERs with inverter interfaces and diesel generators in an islanded microgrid are presented using small signal analysis. It is found that $f-P$ droop gain of the diesel generator impacts its electromechanical mode heavily. On the other hand, $f-P$ and $V-Q$ droop gains in the inverters have insignificant impacts on the electromechanical mode. However $V-Q$ has a significant impact on other system modes. Time-domain simulation results corroborate the analysis results. The major contributions of this paper include: (i) identification of the stability problem in microgrids with both inverter-based DERs and conventional generators and (ii) investigation of the interaction problem of inverter-based DERs and conventional generators in islanded microgrids.

\section{APPENDIX}

The parameters of the synchronous generator for the diesel engines are listed in Table IV.

TABLE IV

SYNCHRONOUS GENERATOR PARAMETERS IN PER UNIT

\begin{tabular}{cc}
\hline Combined inertia of generator and turbine: $\mathrm{H}=5.6 \mathrm{~s}$ \\
$r_{s}=0.003$ \\
$X_{l s}=0.19$ \\
$X_{q}=1.8$ & $X_{d}=1.8$ \\
$r_{k q 1}^{\prime}=0.00178$ & $r_{f d}^{\prime}=0.000929$ \\
$X_{l k q 1}^{\prime}=0.8125$ & $X_{l f d}^{\prime}=0.1414$ \\
$r_{k q 2}^{\prime}=0.00841$ & $r_{k d}^{\prime}=0.01334$ \\
$X_{l k q 2}^{\prime}=0.0939$ & $X_{l k d}^{\prime}=0.08125$ \\
\hline
\end{tabular}

The parameters of the diesel engine are listed in Table V.

TABLE V

DIESEL ENGINE PARAMETERS

\begin{tabular}{cc}
\hline$T_{1}=0.01 s$ & $T_{6}=0.0384 \mathrm{~s}$ \\
$T_{2}=0.02 \mathrm{~s}$ & $T_{D}=24 m s$ \\
$T_{3}=0.2 \mathrm{~s}$ & $T_{\max }=1.1$ \\
$T_{4}=0.25 \mathrm{~s}$ & $T_{\min }=1$ \\
$T_{5}=0.009 \mathrm{~s}$ & \\
\hline
\end{tabular}

The parameters of the diesel generator excitation system are listed in Table VI.

TABLE VI

DIESEL GENERATOR EXCITATION SYSTEM PARAMETERS

\begin{tabular}{c}
$T_{A}=0.005 \mathrm{~s}$ \\
$T_{B}=0.01 \mathrm{~s}$ \\
$T_{E}=0.06 \mathrm{~s}$ \\
$K=0.09$ \\
\hline
\end{tabular}

The parameters of the microgrid network are listed in Table VII.

The parameters in the RL filter in Fig. 4 are listed as follows.

$R=0.41 \Omega$, and $L=5.6 \mathrm{mH}$.

The parameters in the control diagram of an inverter in Fig. 5 are listed as follows.

$C_{P}=K_{p 1}+\frac{K_{i 1}}{s}, C_{Q}=K_{p 1}+\frac{K_{i 1}}{s}$
$C_{i q}=K_{p 2}+\frac{K_{i 2}}{s}, C_{i d}=K_{p 2}+\frac{K_{i 2}}{s}$
TABLE VII

MICROGRID NETWORK PARAMETERS IN PER UNIT

\begin{tabular}{c}
$Z_{26}=0.0031+j 0.0532$ \\
$Z_{12}=0.0031+j 0.0532$ \\
$Z_{23}=0.00227+j 0.00189$ \\
$Z_{24}=0.00275+j 0.00229$ \\
$Z_{25}=0.00075+j 0.00092$ \\
$Z_{T 2}=0.0122+j 0.1462$ \\
$Z_{T 3}=0.1504+j 0.7518$ \\
\hline
\end{tabular}

where $K_{p 1}=1, K_{i 1}=5, K_{p 2}=1$, and $K_{i 2}=100$.

The selection of the proportional and integral gains of the PI controllers is based on the method presented in [24] ( pp. 51). The basic idea is to designate the zero $\left(\frac{K_{i}}{k_{p}}\right)$ of the PI controller provided to cancel the pole of the plant which corresponds to a slow natural response. Therefore the open-loop frequency response will be improved. Further, the proportional gain $K_{p}$ is chosen based on the designated closed loop transfer function time constant.

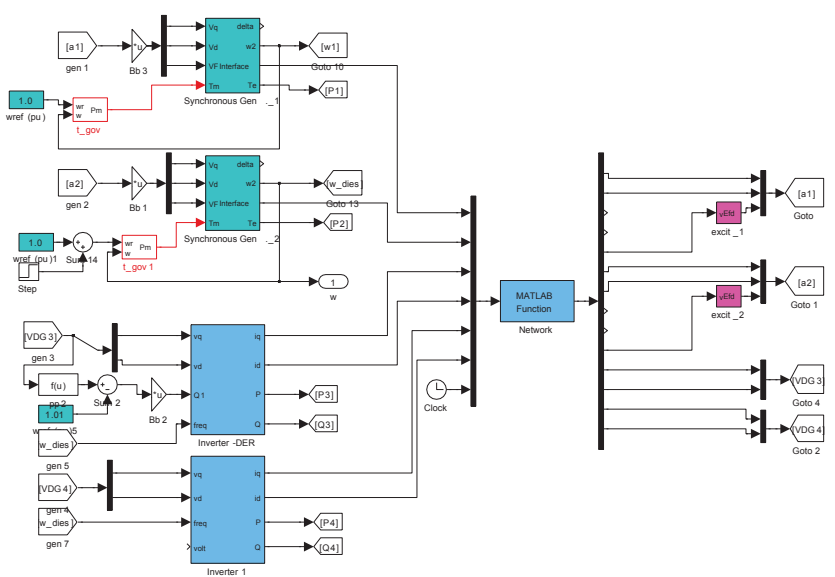

Fig. 14. Simulink diagram of the study system.

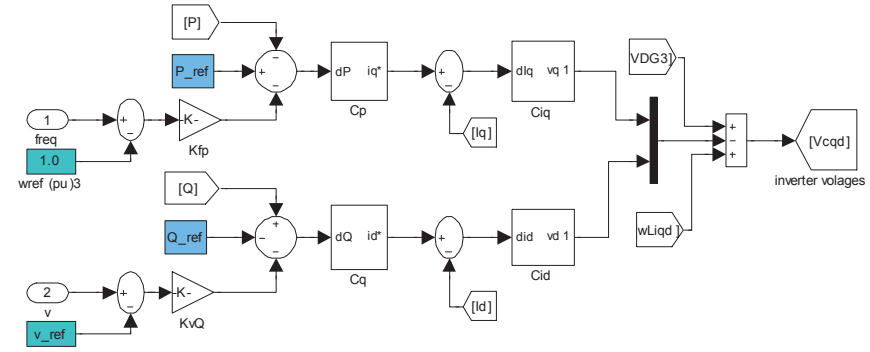

Fig. 15. Control diagram of inverters.

\section{REFERENCES}

[1] E. Barklund, N. Pogaku, M. Prodanovic, C. Hernandez-Aramburo, and T. Green, "Energy management in autonomous microgrid using stabilityconstrained droop control of inverters," IEEE Trans. Power Electron., vol. 23, no. 5, pp. 2346-2352, Sep. 2008.

[2] Y. Mohamed and E. El-Saadany, "Adaptive decentralized droop controller to preserve power sharing stability of paralleled inverters in distributed generation microgrids," IEEE Trans. Power Electron., vol. 23, no. 6, pp. 2806-2816, Nov. 2008. 
[3] F. Peng, Y. Li, and L. M. Tolbert, "Control and protection of power electronics interfaced distributed generation systems in a customerdriven microgrid," in Proceedings of IEEE Power \& Energy Society General Meeting, Calgary, Canada, Jul. 2009.

[4] H. L. Willis and W. G. Scott, Distributed Power Generation: Planning and Evaluation. New York. Basel: Marcel Dekker, Inc., 2000.

[5] S. Krishnamurthy, T. Jahns, and R. Lasseter, "The operation of diesel gensets in a CERTS microgrid," in Proceedings of IEEE Power \& Energy Society General Meeting, Pittsburgh, PA, Jul. 2008.

[6] F. Katiraei and M. Iravani, "Power management strategies for a microgrid with multiple distributed generation units," IEEE Trans. Power Syst., vol. 21, no. 4, pp. 1821-1831, Nov. 2006.

[7] P. Kundur, Power System Stability and Control. McGraw Hill, 1994.

[8] K. D. Brabandere, B. Bolsens, J. D. Keybus, A. Woyte, J. Driesen, and R. Belmans, "A voltage and frequency droop control method for parallel inverters," IEEE Trans. Power Electron., vol. 22, no. 2, pp. 613-625, Mar. 2007.

[9] S. V. Iyer, M. N. Belur, and M. C. Chandorkar, "A generalized computational method to determine stability of a multi inverter microgrid," to appear, IEEE trans. Power Electron.

[10] C. G.-A. J. D. A. Diaz, G.; Gonzalez-Moran, "Scheduling of droop coefficients for frequency and voltage regulation in isolated microgrids," IEEE Trans. Power Syst., vol. 25, no. 1, pp. 489-496, Feb. 2010.

[11] R. Majumder, G. Ledwich, A. Ghosh, S. Chakrabarti, and F. Zare, "Droop control of converter-interfaced microsources in rural distributed generation," IEEE Trans. Power Del., vol. 25, no. 4, pp. 2768-2778, Oct. 2004.

[12] R. Majumder, B. Chaudhuri, A. Ghosh, G. Ledwich, and F. Zare, "Improvement of stability and load sharing in an autonomous microgrid using supplementary droop control loop," IEEE Trans. Power Syst., 2010.

[13] D.-J. Lee and L. Wang, "Small-signal stability analysis of an autonomous hybrid renewable energy power generation/energy storage system part i: time-domain simulations," IEEE Trans. Energy Convers., vol. 23, no. 1, pp. 311-320, Mar. 2008.

[14] F. Katiraei, M. Iravani, and P. Lehn, "Micro-grid autonomous operation during and subsequent to islanding process," IEEE Trans. Power Del., vol. 20, no. 1, pp. 248-257, Jan. 2005.

[15] _ - "Small-signal dynamic model of a micro-grid including conventional and electronically interfaced distributed resources," IET Gener. Transm. Distrib., vol. 1, no. 3, pp. 369-378, May 2007.

[16] "IEEE recommended practice for industrial and commercial power system analysis," IEEE Standard 399, Tech. Rep.

[17] K. Yeager and J. Willis, "Modeling of emergency diesel generators in an 800 megawatt nuclear power plant," IEEE Trans. Power Syst., vol. 8, no. 3, pp. 433-441, Sep. 2003.

[18] M. Rahman, A. Osheiba, T. Radwan, and E. Abdin, "Modeling and controller design of an isolated diesel engine permanent magnet synchronous generator," IEEE Trans. Energy Convers., vol. 11, no. 2, pp. 324-330, Jun. 1996

[19] M. Klein, G. Rogers, and P. Kundur, "A fundamental study of interarea oscillations in power systems," IEEE Trans. Power Syst., vol. 6, pp. 914-921, Aug. 1991.

[20] V. Blasko and V. Kaura, "A new mathematical model and control of a three-phase ac-dc voltage source converter," IEEE Trans. Power Electron., vol. 12, no. 2, pp. 116-123, Jan. 1997.

[21] Z. Jiang and X. Yu, "Active power - voltage control scheme for islanding operation of inverter-interfaced microgrids," Proc. of IEEE Power Engineering Society General Meeting, Jul. 2009.

[22] Z. Miao, "Modeling and dynamic stability of distributed generations," Ph.D. dissertation, West Vriginia University, 2002.

[23] J. Ekanayake and N. Jenkins, "Comparison of response of doubly fed and fixed-speed induction generator wind turbines to changes in network frequency," IEEE Trans. Energy Convers., vol. 19, pp. 800-802, Dec. 2004.

[24] A. Yazdani and R. Iravani, Voltage-Sourced Converters in Power Systems. Wiley, 2010.

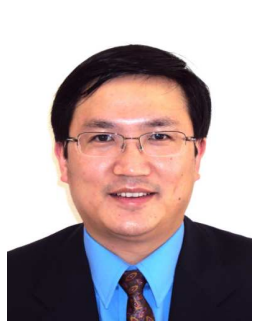

Zhixin Miao (S'00, M'03, SM'09) received his BSEE from Huazhong University of Science \& Technology, Wuhan, China, in 1992. He received his MSEE from the graduate school of Nanjing Automation Research Institute in 1997 and Ph.D. in Electrical Engineering from West Virginia University in 2002 . He is currently with University of South Florida. Prior to joining USF in 2009, he was with the transmission asset management department in Midwest ISO, St. Paul, Minnesota from 2002 to 2009. His research interests include power system stability, microgrid and renewable energy.

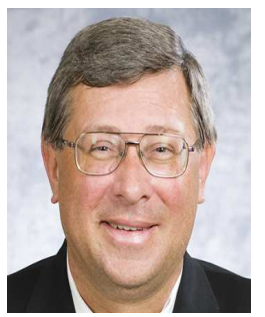

Alexander Domijan, Jr. received the B.S.E.E degree from the University of Miami, Coral Gables, FL, the M.E. degree in electric power engineering from the Rensselaer Polytechnic Institute, Troy, NY, and the Ph.D. degree in electrical engineering from the University of Texas at Arlington.

$\mathrm{He}$ was a member of the faculty at the University of Florida. Currently, he is a Professor with the Department of Electrical Engineering and Director of the Power Quality Laboratory and Distributed Energy Laboratory, University of South Florida, Tampa. He has been a consultant with many corporations. His research areas are PQ and electricity metering, flexible ac transmission systems (FACTS), and customer power and Flexible, Reliable and Intelligent Electrical eNergy Delivery Systems (FRIENDS).

Dr. Domijan is the Editor-in-Chief for the International Journal of Power and Energy Systems.

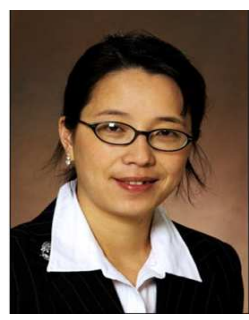

Lingling Fan is an assistant professor in University of South Florida (Tampa, FL) since Aug. 2009. She received the BS, MS degrees in electrical engineering from Southeast University, Nanjing, China, in 1994 and 1997, respectively. She received Ph.D. degree in electrical engineering from West Virginia University in 2001. She was a senior engineer in transmission asset management department in Midwest ISO, St. Paul, Minnesota (2001-2007) and an assistant professor in North Dakota State University (2007-2009). Her research interests include modeling and control of energy systems, large-scale power systems planning and operation. 